Brodman, K., Erdmann, A. J., Lorge, I., Gershenson, C. P., and Wolff, H. G. (1952). Fournal of Clinical Psychology, 8, 119, 289.

Brodman, K., Erdmann, A. J., Lorge, I., Deutschberger, J., and Wolff, H. G. (1954). American fournal Psychiatry, 111, 37.

Brodman, K., Erdmann, A. J., and Wolff, H. G. (1956). Manual for the Cornell Medical Index Health Questionaire, New York.

Brown, A. C., and Fry, J. (1962). Fournal of Psychosomatic Research, 6, 185. Brown, D. G. (1967). Fournal of Psychosomatic Research, 11, 27.

Brown, D. G. (1970). A Study of the Significance of Psychological Symptoms in Eczema. Unpublished M.D. thesis, University of Leeds.

Couper, L., and Twiston Davies, J. H. (1954). British Encyclopaedia of Medical Practice, Interim Supplement No. 144. London, Butterworth. Culpan, R., and Davies, B. M. (1960). Comprehensive Psychiatry, 1, 228.
Culpan, R., Davies, B. M., and Oppenheim, A. N. (1960). British Medical Fournal, 1, 855 .

Edwards, A. L. (1965). Experimental Design in Psychological Research, p. 53. New York, Holt, Rinehart and Winston.

Greenhouse, S. W., and Geisser, S. (1959). Psychometrika, 24, 95.

Hill, J. D. N. (1965). In Skin Diseases in General Practice, ed. F. R. Bettley. London, Eyre and Spottiswoode.

Jacobson, E. (1938). Progressive Relation. Chicago, University of Chicago Press.

Lester, E. P., Wittkower, E. D., Kalz, F., and Azuna, H. (1962). American Fournal Psvchiatrv, 119, 136.

Pillsbury, D. M., Shelley, W. B., and Kligman, A. M., (1956). Dermatology, Saunders, London.

Privat, Y. (1964). Gazette Médicale de France, 71, 1863.

Privat, Y. (1964). Gazette Médicale de France, 71, 1863.
Williams, D. H. (1951). Archives of Dermatology and Syphilology, 63, 545.

\title{
Reliability of Patient Monitoring Apparatus
}

\author{
T. R. EVANS， T. J. H. CLARK
}

British Medical fournal, 1971, 2, 734-736

\section{Summary}

Assessment of a patient monitoring system used in a general medical intensive care ward showed that the blood pressure subsystem was unreliable. The method used in this study is discussed in relation to the clinical requirements of patient monitoring apparatus.

\section{Introduction}

Most clinicians find that patient monitoring devices which seem accurate and easily operated in a research laboratory are less valuable in clinical practice, where they must be reliable as well as accurate. Studies which test only accuracy and reproducibility are therefore inadequate, and we have developed a simple system for estimating the long-term reliability of a monitoring device in a hospital ward. The present trial was carried out in a general medical intensive care unit, and the equipment used was the T.E.M. series II Monitron. The reliability of each subsystem was calculated, and we suggest what should be acceptable levels of reliability for monitoring systems used in intensive care wards.

\section{Materials and Methods}

The Monitron system of patient monitoring has been described elsewhere (Wolff, 1966; Rawles and Crockett, 1969). The series II Monitron is designed to measure respiratory and pulse rates, temperature, and indirect blood pressure. In measuring blood pressure use is made of the phase-shift principle. (De Dobbeleer, 1965), systolic and diastolic blood pressures being detected by an arm-cuff system. An attached printer unit automatically prints out the results at adjustable preset intervals. We have been using series II Monitron apparatus in the medical intensive care unit of Guy's Hospital (Clark et al., 1971) as part of the clinical trials conducted by the Department of Health and Social Security and the Medical Research Council. It was decided not to study the system for measuring respiratory rate, because we had found that most patients tolerated the nasal transducer poorly.

Guy's Hospital, London S.E.1

T. R. EVANS, M.B., M.R.C.P., Research Assistant, Medical Research Council T. J. H. CLARK, M.D., M.R.C.P., Consultant Physician
The pulse rate was recorded from the E.C.G. signal from three chest electrodes, and the temperature measured was that of the skin on the lower back. The E.C.G. signal was displayed on bedside oscilloscopes connected to each individual Monitron unit and on a large-screen slave oscilloscope. The nursing staff were already familiar with the apparatus, but they were given more intensive instruction before the trial began and they had also been routinely using the trial recording sheets for several weeks.

The trial began at 9 a.m. on 1 January 1970 and ended at 9 a.m. on 1 April. All patients monitored in the two Monitron beds during the three-month period were included. Special sheets were designed for recording the times of the patients' connexion and disconnexion from the apparatus and for noting a failure in any system and when it was corrected.

Since nurses in an intensive care unit are usually busy, it was thought desirable to have a 24-hour automatic check on the equipment's performance. The printer unit was therefore set to print the results every 15 minutes. Pulse rate and temperature readings were instantaneous values at the moment of printing, but the blood pressure value was that of the last measuring cycle, the blood pressure unit having been adjusted to cycle automatically every 30 minutes. Often, however, when the blood pressure reading on the indicator display unit was obviously inaccurate the nurse would manually initiate another measuring cycle by pressing the appropriate button on the blood pressure unit.

Previous observation had shown that the printer unit printed the displayed results without significant error, and inspection of the printer unit chart would indicate whether a "nonsense reading" was being displayed on the indicator unit. A "nonsense reading" was a printed reading which was most unlikely to have been correct. For example, all skin temperatures below $33^{\circ} \mathrm{C}$ were regarded as "nonsense readings," since experience had shown that such a reading meant that the termistor probe had become totally or partially detached from the skin. From the blood pressure record it was simple to see when either the systolic or the diastolic blood pressure had not been recorded. Assessment of printed errors of pulse rate was more difficult, since the pulse rate changes more rapidly than other measurements, but records of grossly abnormal pulse rates which in retrospect were known to be false were penalized, as were deviations of more than 10 beats a minute when a patient was being paced with a fixed-rate pacemaker or on continuous demand.

The criteria used to record printed errors were also used by nurses to record failure of a subsystem, and monitoring hours were recorded as unreliable when a fault had been recorded on the trial chart by a nurse or when two or more printed errors were noted within the space of one hour. Errors 
noted by nursing staff were less common and usually lasted most of a monitoring hour, whereas about $75 \%$ of errors lasted for much less than an hour and were found by examination of the printed record. Thus the basic unit of measure was the monitoring hour $(\mathrm{MH})$. One monitoring hour was recorded when one subsystem of the monitoring unit was attached to a patient for one hour.

Three further definitions were used:

Utilized Patient Monitoring.-This equalled the number of hours during which a patient was attached to the unit.

Reliable Patient Monitoring.-This equalled the number of hours during which a patient was reliably monitored.

Coefficient of Monitoring Reliability. - The percentage of the time that the monitoring system functioned reliably when in use.

\section{Results}

There were 30 admissions to bed 1 and 36 to bed 2. Three patients were admitted twice to the same bed and one was admitted twice to the same bed and once to the other bed.

The individual period of monitoring in any one admission varied from 30 minutes to 204 hours 30 minutes, but only three patients were monitored for less than six hours. All three had a fatal cardiac arrest within this time. There were 38 cases of myocardial infarction, six of acute self-poisoning, 17 of various cardiovascular conditions such as arrhythmias not associated with myocardial infarction, including heart block requiring artificial pacemaking, and five of general medical conditions.

The trial extended over 2,160 hours, which gave a total maximum available monitoring time for the two Monitron units of 4,320 MH. Of these, 3,216 $\mathrm{MH}$ were utilized patient monitoring. The average monitoring time per patient was 48.7 $\mathrm{MH}$, and the utilization of monitoring equipment was $74 \cdot 4 \%$.

\section{INDIVIDUAL SUBSYSTEMS}

Pulse Rate.-Utilized patient monitoring=3,216 MH. Reliable patient monitoring $=2,893 \mathrm{MH}$. Coefficient of monitoring reliability $=90 \%$.

Indirect Blood Pressure.-Utilized patient monitoring =3,216 MH. Reliable patient monitoring $=1,575 \mathrm{MH}$. Coefficient of monitoring reliability $=49 \%$.

Temperature.-Temperature probes were unavailable for $590.5 \mathrm{MH}$, since those in use had been broken and replacements were not immediately available. Utilized patient monitoring $=2,624 \mathrm{MH}$. Reliable patient monitoring $=2,347 \mathrm{MH}$. Coefficient of monitoring reliability $=89 \%$.

\section{Discussion}

The assessment was rigorous because it exposed the equipment to the great variety of clinical situations that may arise during more than 3,000 hours of monitoring. We think the results are relevant, because the trial was long and, moreover, the equipment had already been in use for over one year, so that nursing staff were familiar with it. The degree of reliability achieved was in fact higher than could have been reasonably expected in other units short of experienced nursing staff and without technical facilities available. Unfortunately, it is in the latter situation that it had been hoped mechanized monitoring could make a substantial contribution to patient care. It is also important to realize that "nonsense readings" were used as a major criterion of unreliability and therefore if the nurse did not detect other significant errors the subsystems could not be penalized. Any bias is therefore in the direction of underdetection of errors. For example, the results indicate that at best the indirect blood pressure unit was functioning for only $49 \%$ of the available time in a manner that could be considered satisfactory.

It is also significant that the problems with the pulse rate and temperature channels were solved by the hospital medical and technical staff, whereas, owing to its inherent technical complexity, several visits had to be made by the manufacturer's engineer to service the indirect blood pressure unit. On two occasions a blood pressure power unit required factory repair, but on one occasion a spare unit was loaned by the firm. This indicates the need for carrying spare components. While it was too costly to carry spare blood pressure power units, the delay in replacing the temperature probes was partly because we failed to stock spares and partly because an imported component of the probes was unavailable. Manufacturers of monitoring equipment should be prepared to hire or loan the more expensive components whenever repairs are necessary.

Rawles and Crockett (1969) reported their experience with a series I Monitron in a general medical ward over a period of 631 patient-days. They analysed the number of technical faults, but we thought that in an intensive care unit it was particularly important to know exactly how much time was lost. The patient-day was thought to be too large a unit of time for our purposes, hence the use of the monitoring hour. The mean duration of monitoring in Rawles's series was 5.9 days, with a range of from a few hours to 29 days. In our series the mean duration of monitoring was 48.7 hours, with a range of from 30 minutes to $\mathbf{8 . 5}$ days. As Crockett and Barr (1965) pointed out, much of intensive care is intensive observation, and therefore the reliability of the monitoring systems must be very high.

The lack of reliability of the blood pressure unit at any time might have been caused by such factors as loose cuff application, movement of the patient's arm during the measuring cycle, removal of the bulky cuff to facilitate nursing procedures, or that the cuff could not be applied because the patient had a transvenous pacing catheter inserted in one arm and an infusion cannula in the other. These situations might not be considered to indicate primary equipment failure, but to the clinician they have the same significance-the failure of monitoring apparatus in everyday clinical situations. With nursing staff carefully instructed in the use of the equipment, who appreciated the purpose of the trial, and who took professional pride in trying to keep the equipment working it was impossible to achieve more than $49 \%$ reliability with the blood pressure module. In practical terms this meant that for over half the time for which patients were being monitored no satisfactory blood pressure readings were available.

Even in the cases of uncomplicated myocardial infarctiongenerally the most co-operative and amenable to monitoring procedures-the performance of the blood pressure unit under normotensive conditions varied from nearly maximum to zero reliability. In fact, when accurate blood pressure readings were vital nurses would rarely rely on the module and often discarded it completely when the readings were inaccurate.

It is important with patient monitoring equipment to consider whether a measurement is one the nurse herself can makefor example, indirect blood pressure-or whether the use of electronic equipment-for example, to obtain an E.C.G. signal-is essential. The attitude of the nurse towards the two types of equipment is very different. She is prepared to accept a few problems in order that an essential physiological process may be monitored, but when she knows the machine is making only a measurement that she herself can make she tends to be more hostile when it is unreliable.

From the present study we have concluded that of the Monitron subsystems available only those for measuring temperature and heart rate are satisfactory, and that a new method of indirect blood pressure measurement is needed. In the meantime blood pressure may have to be monitored by an intra- 
arterial line in selected cases, but in our opinion it is neither necessary nor justifiable to insert routinely intra-arterial lines in all patients admitted to a general medical intensive care unit.

Flexibility is the essence of modular monitoring apparatus. It allows the clinician to decide which physiological variables he wishes to monitor, and he should never regard such a system as a "panindicator" unit. Therefore each separate system must be reliable in its own right, and reliability in the clinical environment can be assessed only by intensive study in a clinical situation. The technique we used seems to give a useful indication of whether a particular system is suitable for routine clinical use. Moreover, we would suggest that to be of value in hospital conditions an automatic monitor needs to be at least $90 \%$ reliable.
We would like to acknowledge the unstinted co-operation of the nursing staff of Starling Ward; Mr. A. J. Solman, of the National Institute for Medical Research; and Mr. A. S. Velate, of the T.E.M. Engineering Company.

This work was supported by the Medical Research Council.

\section{References}

Clark, T. J. H., Collins, J. V., Evans, T. R., and Tweedily, K. (1971). British Medical fournal, 1, 158

Crockett, A. S., and Barr, A. (1965). British Medical fournal, 2, 1173.

De Dobbeleer, jun., G. D. P. (1965). World Medical Electronics and Instrumentation, 3, 1222

Rawles, J. M., and Crockett, G. S. (1969). British Medical fournal, 3, 707.

Wolff, H. S. (1966). British Hospital fournal and Social Service Review, 76, 1991 .

\title{
Monitoring Cerebral Function: Clinical Experience with New Device for Continuous Recording of Electrical Activity of Brain
}

\author{
PAMELA F. PRIOR, D. E. MAYNARD, P. C. SHEAFF， B. R. SIMPSON, L. STRUNIN, \\ E. J. M. WEAVER, D. F. SCOTT.
}

British Medical fournal, 1971, 2, 736-738

\begin{abstract}
Summary
A device, the cerebral function monitor, provides a continuous record of the electrical activity of the brain occurring at frequencies from 2 to $15 \mathrm{~Hz}$. It is relatively cheap, portable, and easy to use and interpret. The apparatus has proved of value in three circumstances: firstly, when the cerebral circulation is likely to be vulnerable during open heart surgery; secondly, as a measure of recovery or deterioration following brain damage or drug overdose; and thirdly, where information about more physiological changes in cerebral function is required, for instance when testing anaesthetic and hypnotic drugs.
\end{abstract}

\section{Introduction}

Continuous monitoring of cerebral activity may be of particular value in two situations. Firstly, when the cerebral circulation is likely to be vulnerable, for instance during open cardiac surgery, and secondly, as a measure of recovery or deterioration following brain damage or in drug induced coma. The use of electroencephalographic (E.E.G.) apparatus for examining the electrical activity of the brain for long periods is complex and

The London Hospital, London E1 1BB

PAMELA F. PRIOR, M.B., Medical Assistant, E.E.G. Department D. E. MAYNARD, M.PHIL., Research Engineer, E.E.G. Departmen P. C. SHEAFF, M.R.C.P., Registrar, E.E.G. Department (At present Senior Registrar, Department of Clinical Neurophysiology, St. Bartholomew's Hospital, London EC1A 7BE)

B. R. SIMPSON, D.PHIL., F.F.A. R.C.S., Professor, Anaesthetics Unit L. STRUNIN, M.B., F.F.A. R.C.S., Senior Lecturer and Consultant Anaesthetist, Anaesthetics Unit

E. J. M. WEAVER, F.R.C.s., Surgeon, Department of Cardiothoracic Surgery

D. F. SCOTT, M.R.C.P., D.P.M., Consultant-in-Charge, E.E.G. Department costly in terms of specialized staff and apparatus (Feldman and Ellis, 1967; Haider et al., 1971). Equipment aimed at monitoring brain activity has been part of a system for providing servo-anaesthesia (Bickford, 1949) or used to indicate the depth of anaesthesia (Efuni et al., 1965); none has been primarily used for providing information about the state of cerebral activity.

A cerebral function monitor (C.F.M.) has recently been introduced (Maynard et al., 1969) as a result of experience at the London Hospital of E.E.G. recording in the operating theatre during cardiac surgery (Fischer-Williams and Cooper, 1964) and in the intensive therapy unit (Prior and Volavka, 1968; Binnie et al., 1970). The C.F.M. is designed to obtain a simple, continuous assessment of cerebral function over long periods without the need for skilled attention. This communication reports our use of the C.F.M. in patients following cardiorespiratory arrest and during open heart surgery. Studies have been undertaken in close parallel with conventional E.E.G. recordings; these have been carried out every 24 to 48 hours on patients in the intensive therapy unit and continuously throughout most of the operations on the patients undergoing cardiac surgery. On occasions the C.F.M. has been used alone during cardiac surgery to see whether it is of value to the anaesthetist without the aid of an E.E.G. technician or an electroencephalographer.

\section{Methods and Materials}

The technical details of the C.F.M.* have been described (Maynard et al., 1969). Briefly, cerebral activity is picked up by two conventional silver and silver chloride electrodes attached with collodion to the parietal regions on both sides of the head after preliminary separation of the hair and cleansing of the scalp. This site was chosen to reduce muscle and movement artefact and to minimize interference during nursing procedures. The output of the monitor is seen as two traces on a hot wire recorder. The first trace gives a continuous record of the

*Available from Devices Limited, Welwyn Garden City, Herts. 